\title{
The Diagnosis of Acute Pulmonary Embolism
}

\author{
Ebtesam Islam MD PhD, Victor J Test MD
}

\section{Abstract}

This paper reviews the most current literature on the diagnosis of pulmonary thromboembolism. The epidemiology and symptomology of this disorder, including common symptoms such as fever, chest pain, dyspnea, edema, and syncope, are reviewed. The utility of basic and easily available testing, such as electrocardiography and chest radiography, is evaluated. The literature on determining the pretest probability of venous thromboembolism with scoring systems, such as the Wells Score, the Geneva Scoring System, and the Pulmonary Embolism Rule Out Criteria, is appraised. As the evaluation of pulmonary embolism has evolved, multiple imaging techniques has been developed and studied. Ultrasonography, computed tomography with angiography, magnetic resonance angiography, ventilation perfusion lung scanning, and SPECT ventilationperfusion lung imaging are discussed. In conclusion, the diagnosis of pulmonary embolism remains complicated. Clinical suspicion and stratification should guide a diagnostic strategy for the comprehensive evaluation and diagnosis of patients with this disorder.

Keywords: pulmonary embolism, deep venous thrombosis, diagnosis, CT angiography, ventilation-perfusion scans, clinical decision rules

Pulmonary embolism (PE) is a leading cause of morbidity and mortality in the United States, and between $5 \%$ and $10 \%$ of hospital deaths are attributable to PE. ${ }^{1}$ From 1998 to 2005 , the number of patients discharged from United States hospitals with a diagnosis of pulmonary embolism increased from 126,546 to $229,637 .{ }^{1}$ Over this period, the hospital case fatality rate decreased from $12.3 \%$ to $8.2 \%(p<0.001){ }^{1}$ The length of stay decreased, but hospital charges for these patients increased nearly $100 \%(p<0.001)$. In

Corresponding author: Victor Test

Contact Information: Victor.test@ttuhsc.edu

DOI: 10.12746/swrccc 2014.0208.099 the United States, approximately 100,000 to 200,000 deaths occur in over 600,000 episodes of pulmonary embolism per year. $^{2-6}$ An overwhelming majority of these deaths occur when the disease is under-recognized or misdiagnosed and ultimately discovered on autopsy. ${ }^{7,8}$ With the correct diagnosis and effective treatment, the risk of death diminishes dramatically. ${ }^{9}$ As expected, patients who present with shock have the highest mortality from PE. Unfortunately, even with myriad diagnostic tests and treatment options available, PE is common, lethal, and underdiagnosed. ${ }^{1,10}$

Pulmonary embolism can present along a spectrum from the asymptomatic individual incidentally diagnosed to the patient presenting with cardio- 
genic shock. ${ }^{11}$ Thus the diagnosis of acute PE is ultimately guided by the clinician's index of suspicion for the disease and augmented by diagnostic tests. PE is closely linked with deep venous thrombosis (DVT) and should be considered a different manifestation of the same disorder, namely venous thromboembolism (VTE). The recognition of the signs and symptoms of $P E$ is the most important initial diagnostic step. Acareful clinical history and physical examination is crucial to identify the patients at risk and to assess the pretest probability. In a review of the Prospective Investigation of Pulmonary Embolism Diagnosis (PIOPED 1) data, Stein and Henry found that dyspnea was the most common symptom followed by pleuritic chest pain, cough, lower extremity edema, hemoptysis, palpitations, wheezing, and angina-like pain. ${ }^{12}$ Pleuritic chest pain and hemoptysis are more common in patients with pulmonary infarction. ${ }^{12} \mathrm{PE}$ should always be considered in patients with chest pain, dyspnea, hemoptysis, syncope, and palpitations. ${ }^{13}$ The possibility of PE can be subtle with non-specific symptoms and signs, such as tachycardia, tachypnea, and fever. In Stein's study, tachypnea was the most common physical examination finding followed by crackles, tachycardia, and an increased pulmonic heart tone. ${ }^{12}$ Other examination findings in this study included evidence of DVT, fever $>38.5^{\circ} \mathrm{C}$, diaphoresis, wheezing, and a pleural friction rub in $6 \%$ to $14 \%$ of patients. ${ }^{12}$ Lower extremity signs, such as edema, leg and calf tenderness, erythema, venous cords, and Homan's sign, may indicate a DVT. Classically, $90 \%$ of emboli originate from proximal lower extremity DVT. ${ }^{13}$ However, only $48.6 \%$ of patients with a high probability of PE had a DVT. ${ }^{14}$ Upper extremity venous thrombosis and catheter-associated thromboses are additional sources of PE. Due to the nonspecific symptoms of PE and DVT, there can be substantial delays in seeking medical attention and diagnosis once medical attention is sought. ${ }^{15}$

\section{Clinical suspicion and clinical decision rules}

The diagnosis of PE and DVT is dependent upon the clinician's suspicion of the disease. Unfortunately, there are numerous studies that demonstrate failures or delays in diagnosis of PE. ${ }^{2-6,11}$ Further, the morbidity and mortality of VTE increase when the diagnosis is not made. ${ }^{2-6}$ Paradoxically, as the evaluation for VTE has evolved, more patients are undergoing evaluation with imaging for PE, but the diagnostic yield of these tests can be as low as $3.1 \%$ in the absence of clinical prediction rules. ${ }^{16}$ There are numerous risk factors for VTE, including age greater than forty, previous VTE, surgery requiring anesthesia for more than $30 \mathrm{~min}$ utes, prolonged immobilization, stroke, heart failure, malignancy, fractures of the long bones or pelvis, spinal cord injury, obesity, smoking, pregnancy, estrogen therapy, inflammatory bowel disease, and genetic or acquired thrombophilia. Renal failure, nephrotic syndrome, central venous catheterization, COPD, and long distance travel have also been identified as risk factors. ${ }^{11}$ Hip/knee surgery/fracture and spinal cord injury carry the highest risk.

\section{Risk stratification and pretest probability}

Stratifying patients into low risk, moderate risk, or high risk categories can be performed either by empiric assessment and "gestalt" or by the use of structured clinical prediction rules. ${ }^{17}$ Structured clinical prediction rules standardize the approach to pretest assessment of probability and remove the variability of clinical experience found in clinical practice. There are numerous clinical prediction scores, including the Wells Score, Simplified Wells Score, Geneva Score, Revised Geneva Score, Simplified Revised Geneva Score, Miniati Score for Likelihood of Pulmonary Embolism, the Charlotte Rule, and the Hyer Score. ${ }^{18-24}$ The Wells Rules, Simplified Wells Rules, and the Geneva scores are frequently used in clinical trials and have the most validation from clinical studies. ${ }^{18}$ The clinical prediction rules devised by Wells, et al. use a point based system based on historical factors, such as malignancy (1 point), hemoptysis (1 point), previous DVT/PE (1.5 points), immobilization or recent surgery (1.5 points), heart rate $>100$ beats/minute (1.5 points), clinical evidence of DVT (3 points), and absence of equally likely alternative diagnosis (3 points). ${ }^{25}$ In this point system, low ( $<2$ points), intermediate (2-6 points), and high probability (>6 points) 
indicate pretest probability. The simplified Wells rules assigns one point for each criterion and establishes a cut point above and below 4 to distinguish between low pretest probability and intermediate or high pretest probability. ${ }^{25}$ The Pulmonary Embolism Rule Out Criteria (PERC) has been proposed as a method to decrease testing in suspected pulmonary embolism. These criteria use a point system in which each crite- rion is valued at one point, and PE is ruled out if all of the criteria are negative. The PERC score includes the following criteria: age $>55$ years, heart rate $>100$ beats per minute, room air oxygen saturation $<95 \%$, previous episode of VTE, exogenous estrogen, recent surgery, unilateral leg swelling, and hemoptysis. A recent retrospective study found an incidence of $0.5 \%$ in 1020 patients with a negative PERC score. ${ }^{2}$

\section{TABLE 1: Wells scores for pretest probability calculation}

\begin{tabular}{llll}
\hline Wells Criteria & Points & Simplified Wells & Points \\
\hline Clinical Signs of DVT & 3.0 & Clinical Signs of DVT & 1.0 \\
Recent Surgery Immobilization & 1.5 & Recent Surgery Immobilization & 1.0 \\
Heart Rate $>100 \mathrm{bpm}$ & 1.5 & Heart Rate $>100 \mathrm{bpm}$ & 1.0 \\
Previous VTE & 1.5 & Previous VTE & 1.0 \\
Hemoptysis & 1.0 & Hemoptysis & 1.0 \\
Malignancy & 1.0 & Malignancy & 1.0 \\
Alternative Diagnosis less likely than PE & 3.0 & Alternative Diagnosis less likely than PE & 1.0 \\
\hline
\end{tabular}

3-level Wells Score: Low $<2$ points, Intermediate 2-6 points, high $>6$ points. 2-level Wells Score PE Unlikely $\leq 4$ points PE likely $>4$ points. Simplified Wells Score: $\mathrm{PE}$ unlikely $\leq 1$ point, $\mathrm{PE}$ Likely $>1$ point. $\mathrm{BPM}=$ beats per minute, $\mathrm{PE}=$ pulmonary embolism, VTE $=$ venous thromboembolism

\section{TABLE 2: Geneva scores for pretest probability calculation}

\begin{tabular}{llll}
\hline Revised Geneva & Points & Simp Rev Geneva & Points \\
\hline Age $>$ 65 years & 1.0 & Age $>65$ & 1.0 \\
Previous VTE & 3.0 & Previous VTE & 1.0 \\
Surgery or Fracture within 1 month & 2.0 & Surgery or Fracture within one month & 1.0 \\
Active Malignancy & 2.0 & Active Malignancy & 1.0 \\
Heart Rate 75-94 bpm & 3.0 & Heart Rate 75-94 bpm & 1.0 \\
Heart Rate $>95$ bpm & 5.0 & Heart rate $>95 \mathrm{bpm}$ & 1.0 \\
Unilateral Leg Pain & 3.0 & Unilateral leg pain & 1.0 \\
Hemoptysis & 2.0 & Hemoptysis & 1.0 \\
Pain on deep leg palpation & 4.0 & Pain on deep leg palpation & 1.0 \\
\hline
\end{tabular}

Revised Geneva Score: Low PTP 0-3 points, Intermediate PTP 4-10 points, High PTP $>11$ points. Simplified Revised Geneva Score: Low PTP 0-1 point, Intermediate 2-4 points, High 5 points or more.

\section{Diagnostic testing}

Electrocardiogram, chest radiographs, and selected laboratory tests

Routine initial diagnostic testing in the evaluation of a patient with symptoms suggestive of a PE is neither sensitive nor specific. Over the past twenty years, a bewildering number of diagnostic tests, either alone or in combination, has been studied as a means of excluding or confirming the diagnosis of PE. Arterial blood gas testing may demonstrate a respiratory alkalosis or hypoxemia, but arterial blood gas evaluation including the alveolar-arterial 
gradient is neither sensitive nor specific. ${ }^{12,27}$ The chest radiograph often demonstrates nonspecific findings; atelectasis (52-75\%), pleural effusion (26-56\%), pleural based opacities (23-36\%), elevation of the diaphragm, cardiomegaly, and a normal radiograph can be seen. ${ }^{12,13,28} \mathrm{~A}$ large international cooperative registry found that cardiomegaly on chest radiograph was the most common finding in pulmonary embolism followed by normal radiograph, pleural effusion, elevated diaphragm, atelectasis, and pulmonary artery enlargement. ${ }^{29}$ The electrocardiogram (ECG) most commonly reveals sinus tachycardia or a normal electrocardiogram. The classic finding S1Q3T3 denotes right ventricular strain and is rarely seen in PE. ${ }^{12,30,31}$ In Stein's evaluation of PIOPED 1, patients with PE had abnormal ECG between $39 \%$ of the time. The ECG was abnormal in $10 \%$ of patients who presented with isolated dyspnea compared to $54 \%$ of patients with pulmonary infarction and $80 \%$ of patients with circulatory collapse. The most common findings were nonspecific ST segment or T wave changes and were found in $44 \%$ of patients. ${ }^{12}$ Other ECG findings included sinus tachycardia, non-specific ST-T wave changes, and right bundle branch block. ${ }^{12}$ Brain natriuretic peptide (BNP) levels are higher in patients with $P E$ than those without PE; however, it is nonspecific and insensitive. ${ }^{32}$ In a case-control study of patients with hemodynamically stable PE, BNP had a sensitivity of $60 \%$ and specificity of only $62 \% .{ }^{32}$ Serum troponin I and troponin $\mathrm{T}$ are elevated in PE, but are not useful in diagnosis. ${ }^{33}$ They may be useful for risk stratification of patients with anatomically large PE. ${ }^{33}$ Heart type fatty acid binding protein (H-FABP) is a highly sensitive marker for myocardial infarction that can be used as a predictor for outcome in acute PE. ${ }^{34}$ The 30-day mortality for acute PE using the H-FABP had a $98 \%$ sensitivity and $77 \%$ specificity. ${ }^{34}$

\section{D-dimer testing}

The D-dimer is a cross-linked fibrin degradation product. It is elevated in active thrombosis and useful in identifying patients with possible PE. It has an excellent sensitivity in the evaluation of VTE but poor specificity. Conditions such as increasing age, malignancy, hospitalization, and previous DVT adversely affect the specificity of the D-dimer. ${ }^{35,36}$ The different assays have a wide range in sensitivities. ${ }^{25,37}$ The enzyme linked immunoabsorbent assay (ELISA) $D$-dimer is most useful in ruling out the diagnosis in outpatients who have a low pretest probability of PE. ${ }^{37}$ In a large meta-analysis of over 7000 patients, the negative likelihood ratio of an ELISA (enzyme linked immunoabsorbent assay) was 0.13 , and for a rapid ELISA it was $0.13 .^{38}$ In this analysis, whole blood and less sensitive qualitative assays had negative likelihood ratios that were not as useful in ruling out VTE. ${ }^{38}$ D-dimer increases with age, reducing the ability to rule out $\mathrm{PE}$ in elderly patients with the cutoff value usually being $500 \mu \mathrm{g} / \mathrm{L}$. However, a recent study with age adjusted D-dimer (patient's age multiplied by 10 if age greater than 50 years), PE could be ruled out in a larger number of patients. ${ }^{39}$

When combined with structured clinical decision rules, the D-dimer can be very useful in excluding the diagnosis of pulmonary embolism. ${ }^{16,19,20,40-44}$ In patients who are at low risk based on the scoring systems listed above, the incidence of pulmonary embolism at three months in the setting of a negative highly sensitive D-dimer assay is extremely low. ${ }^{37}$ The combination of the Wells rule plus a negative D-dimer carried a risk of VTE during a three month follow-up period of $0-1.1 \%$ in two studies. ${ }^{43,44}$ In general, intermediate and high-risk patients should receive diagnostic imaging.

\section{Medical Imaging}

\section{Compression ultrasound}

Compression ultrasound of the extremities is commonly used in the evaluation of VTE. It is readily available, has no radiographic contrast, and is noninvasive. The compression ultrasound has excellent sensitivity and specificity in symptomatic patients with proximal DVT. The estimated sensitivity is $89-96 \%$, and the specificity is $94-99 \% .{ }^{41}$ In the evaluation of patients with suspected PE, a compression ultrasound is positive in $10-14 \%$ of patients when used as the initial diagnostic test. ${ }^{45,46}$ When positive, it may limit the need for additional testing. ${ }^{18}$ The yield of ultrasound 
can be increased when the patient has symptoms or signs of proximal DVT. However, compression ultrasound cannot be used in isolation for the exclusion of $\mathrm{PE}$ as over $50 \%$ of patients with PE will have a negative ultrasound of the extremity veins. ${ }^{46,47}$

The anatomically based techniques used to image PE include ventilation and perfusion scintiphotography, contrast enhanced helical CT scanning, pulmonary angiography, echocardiography, and contrast-enhanced MRI. Each study has its own specific advantages and limitations.

\section{Ventilation perfusion (V/Q) lung scanning}

V/Q scanning was the imaging modality of choice until recent years. It is highly dependent on the baseline chest radiograph. ${ }^{48}$ Indeterminate results were obtained in up to $54 \%$ of patients in one study. ${ }^{49} \mathrm{~A}$ more recent study suggested that with the Prospective Investigative Study of Acute Pulmonary Embolism Diagnosis (PISAPED) criteria the sensitivity of a PEpresent scan $(80.4 \%)$ and the specificity of a PE-absent scan (96.6\%) were comparable to PIOPED data, but the number of non-diagnostic scans decreased from $20.6 \%$ using PIOPED criteria to $0 \%$ using PISAPED criteria. ${ }^{50} \mathrm{~V} / \mathrm{Q}$ scanning does not require iodinated contrast and has significantly lower radiation exposure than CT scanning. ${ }^{49}$ As documented in the PIOPED 1 study, a normal V/Q scan virtually rules out a $P E$ in the patient with a low to intermediate pre-test probability. ${ }^{48}$ Patients with high clinical probability of PE and high probability V/Q scans had a 95\% likelihood of having PE.48 Patients with low clinical probability of PE and low probability V/Q scans had only a $4 \%$ likelihood of having $P E$. A recent prospective study demonstrated that a diagnostic protocol using clinical prediction rules and V/Q scanning has a high diagnostic yield when combined with compression ultrasound. ${ }^{42}$ A diagnosis of VTE was established in $76 \%$ of patients and only $11 \%$ of patients required CT angiography. ${ }^{42} \mathrm{~V} / \mathrm{Q}$ scanning should be considered as first-line imaging with a normal chest radiograph, in pre-menopausal women, pregnant women, patients with renal insufficiency, and patients with contrast allergy. ${ }^{19,48} \mathrm{~A} V / \mathrm{Q}$ scan is still quite useful but may be less available due to the increasing use of CT scanning as the preferred choice in many centers. Single photon emission computed tomography (SPECT) is becoming more popular due to its ability to image in three dimensions as opposed to two dimensional imaging for lungs. ${ }^{49}$ SPECT provides a more accurate size estimate and location of perfusion defects in subsegments. ${ }^{48}$ With its higher image intensity contrast, it is more sensitive than planar perfusion scanning for identifying obstructed segments in chronic thromboembolic pulmonary hypertension. ${ }^{50}$

\section{CT pulmonary angiography and venography}

The advent of the multislice, multidetector CT scanner has made a substantial impact on the diagnosis of PE. The PIOPED II study compared multidetector CT angiogram (CTA) using $1.25 \mathrm{~mm}$ cuts with multidetector CTA with venography (CTA/CTV)..$^{53}$ The sensitivity of CTA was $83 \%$ with a specificity of $96 \%$ for PE. ${ }^{52}$ The sensitivity of CTA/CTV was $90 \%$, and the specificity was $95 \% .{ }^{53}$ In PIOPED II, the number of nondiagnostic studies was $11 \%$, and when pooled with other trials, non-diagnostic studies occurred in $6 \%$ of patients. ${ }^{19,53}$ The CT PIOPED II study demonstrated excellent negative predictive value (99.1\%) and had good positive predictive value. ${ }^{53}$ CTV did not significantly alter posttest probability. ${ }^{53}$ It is clear from this study that the concept of pretest probability is still necessary in the assessment for PE. ${ }^{53}$ Most centers do not use combination CTA and CTV, and we are often forced to use duplex ultrasonography to augment the negative CT to rule out a significant PE for patients with high pretest likelihood of VTE. ${ }^{19,53}$ The CTA also has the benefit of evaluating lymph nodes and the lung parenchyma. The crucial disadvantages include the need to obtain high quality scans with well timed boluses of contrast to obtain an optimal scan and the use of intravenous contrast. lodinated contrast can cause renal dysfunction and allergic reactions. CTA typically uses more contrast than standard pulmonary angiography. The use of CT scans has increased at an average of rate of $28.1 \%$ per year, and this, of course, increases radiation exposure to patients. ${ }^{54} \quad$ Exposure to five to six chest CT scans is equivalent to an effective dose in atomic bomb 
survivors of $40 \mathrm{mSv}$. The most cancer susceptible organs are thyroid, breast, and lungs; the exposures from CTA scans is 100 to 400 times more than a two view chest radiograph. ${ }^{55}$

\section{Pulmonary angiography}

Pulmonary angiography is considered the standard imaging procedure but in general has fallen out of use in most centers due to its perceived risks and the increased use of CT scanning. ${ }^{56}$ It carries a significant risk in patients with acute PE. ${ }^{57}$ Moreover, in comparison to studies with CTA, it appears to have a lower sensitivity than CTA. ${ }^{56,58-60}$

\section{Magnetic resonance imaging}

PIOPED III was a multicenter study designed to assess the sensitivity and specificity of magnetic resonance angiography (MRA) alone or with magnetic resonance venography for diagnosing pulmonary embolus. Unfortunately, MRA was technically inadequate in $25 \%$ of patients. ${ }^{61}$ In patients with technically adequate images, MRA was $78 \%$ sensitive and 99\% specific. ${ }^{61}$ However, MRA should be considered only at centers that routinely perform the study and perform it well due to the difficulties in obtaining technically adequate images. MRA should be considered experimental at present. ${ }^{9,61} \mathrm{MRI}$ lacks the resolution, wider availability, and larger clinical experience that CT has attained. ${ }^{60}$ As technology advances, the diagnostic value of each test is likely to evolve.

\section{Thoracic ultrasound}

Multiorgan ultrasonography has also become popular since it decreases the radiation and contrast associated with multidetector CTA. In a multicenter, prospective trial, Nazerian, et al. showed that lung, heart, and leg ultrasound along with D-dimer assays improved the accuracy of diagnosing PE. In addition, it is a quick tool that can be used at the beside in unstable patients. ${ }^{62}$ Ultrasound was considered positive and diagnostic of PE if the sonographers were able to detect subpleural infarcts in the lung, right ventricular dilatation, thrombi in the heart, or the absence of total vein collapse during compression for DVT. ${ }^{62}$ The presence of PE was confirmed by multidetector CTA in this study. Multiorgan sonography had a $90 \%$ sensitivity (higher than any individual sonography) and $86.2 \%$ specificity. ${ }^{62}$

\section{Echocardiography}

Transthoracic echocardiogram is often used to assess chest pain. In acute PE, the echocardiogram is useful to stratify hemodynamically stable patients to determine which patient is at risk for a poor outcome. ${ }^{63-65}$ However, its use in an acute setting is sometimes limited by availability, cost, and interobserver variability in the interpretation of results. The findings of right ventricular dilatation and pulmonary hypertension are poor markers for increased mortality. ${ }^{63-65}$ The echocardiogram may show evidence of

TABLE 3: Studies currently capable of EXCLUDING the diagnosis of embolism at different levels of clinical probability

\begin{tabular}{|c|c|c|c|}
\hline & $\begin{array}{l}\text { Low Clinical } \\
\text { Probability }\end{array}$ & $\begin{array}{l}\text { Intermediate Clinical } \\
\text { Probability }\end{array}$ & $\begin{array}{l}\text { High Clinical } \\
\text { Probability }\end{array}$ \\
\hline Elisa D-dimer & Negative & Negative & ---------- \\
\hline $\begin{array}{l}\text { Ventilation/Perfusion } \\
\text { Scan }\end{array}$ & $\begin{array}{l}\text { Normal or low } \\
\text { probability }\end{array}$ & Normal & Normal \\
\hline $\begin{array}{l}\text { Computed } \\
\text { Tomography }\end{array}$ & Normal & Normal & ---------- \\
\hline Contrast Angiography & Normal & Normal & Normal \\
\hline
\end{tabular}




\section{TABLE 4: Studies currently capable of CONFIRMING the diagnosis of embolism at different levels of clinical probability}

\begin{tabular}{|c|c|c|c|}
\hline & $\begin{array}{l}\text { Low Clinical } \\
\text { Probability }\end{array}$ & $\begin{array}{l}\text { Intermediate Clinical } \\
\text { Probability }\end{array}$ & $\begin{array}{l}\text { High Clinical } \\
\text { Probability }\end{array}$ \\
\hline Elisa D-dimer & ---------- & ---------- & ---------- \\
\hline Duplex Ultrasound & Positive & Positive & Positive \\
\hline $\begin{array}{l}\text { Ventilation/Perfusion } \\
\text { Scan }\end{array}$ & ---------- & High Probability & High Probability \\
\hline $\begin{array}{l}\text { Computed } \\
\text { Tomography }\end{array}$ & Positive & Positive & Positive \\
\hline Contrast Angiography & Positive & Positive & Positive \\
\hline
\end{tabular}

right ventricular strain or overload. In addition, the echocardiogram is often used to identify patients at risk for the development of hemodynamic decompensation. ${ }^{63-65}$ Transesophageal echocardiography can image the proximal pulmonary arteries and confirm a diagnosis of proximal massive PE, but it is unable to visualize the intermediate branches of the pulmonary artery. ${ }^{64}$

\section{Summary}

Multiple tests are available to aid in the diagnosis of pulmonary embolus. The diagnosis of PE is guided by suspicion for the disease. The classic signs and symptoms of DVT and PE and the risk factors for VTE are well known. The different clinical prediction models available help stratify risk and demonstrate the broad spectrum of VTE presentations. Interpretation of the diagnostic testing procedures should be guided by the clinical probability for the presence of PE. It is important to definitively rule out the diagnosis in the stable outpatient, but it is equally important to make this diagnosis in patients in cardiogenic shock with massive PE. Multiple diagnostic tests and evaluations may be required for assessment of PE. We must remain vigilant in our efforts to diagnose and treat this deadly disorder.
Author affiliation: Ebtesam Islam is a fellow in pulmonary and critical care medicine at TTUHSC. Victor Test is in the pulmonary and critical care division in the Department of Internal Medicine at TTUHSC.

Received: $10 / 1 / 14$

Accepted: 10/8/14

Reviwer: Kenneth Nugent MD

Published electronically: 10/15/14

Conflict of Interest Disclosures: None

\section{References}

1. Park B, Messina L, Dargon P, et al. Recent Trends in Clinical Outcome and Resource Utilization for Pulmonary Embolism in the United States: Findings from the Nationwide Inpatient Sample. Chest 2009; 136: 983-990.

2. Dalen JE. Pulmonary embolism: what have we learned since Virchow? Natural history, pathophysiology, and diagnosis. Chest 2002; 122:1440-56.

3. Dalen JE, Alpert JS. Natural History of Pulmonary Embolism. Prog Cardiovasc Dis 1975; 17: 257-70.

4. Lilienfeld DE, Chan E, Ehland J, et al. Mortality from pulmonary embolism in the United States: 1962 to 1984. Chest 1990; 98:1067-72

5. Clagett GP, Anderson FA Jr, Heit J, et al. Prevention of venous thromboembolism. Chest 1995; 108(suppl):312S-334S 6. Anderson FA, Wheeler HB, Goldberg RJ. A populationbased perspective of the hospital incidence and case fatality rates of deep venous thrombosis and pulmonary embolism: the Worchester DVT Study. Arch Intern Med 1991; 151:933-938 
7. Ryu JH, Olson EJ, Pellikka PA. Clinical Recognition of Pulmonary Embolism: Problem of Unrecognized and Asymptomatic Cases. Mayo Clin Proc 1998; 73: 873-79. 8. Pulido T, et al. Pulmonary Embolism as a Cause of Death in Patients with Heart Disease: An Autopsy Study. Chest 2006; 129: 1282-87.

9. Carson JL, Kelly MA, Duff A, Weg JG, et al. The clinical course of pulmonary embolism. N Engl J Med 1992; 326:1240-5.

10. Wood KE. Major Pulmonary Embolism: Review of a Pathophysiologic Approach to the Golden Hour of Hemodynamically Significant Pulmonary Embolism. Chest 2002; 121; 877-905.

11. Tapson, VF. Acute Pulmonary Embolism. N Engl J Med 2008; 358:1037-52.

12. Stein PD, Henry JW. Clinical Characteristics of Patients with Acute Pulmonary Embolism Stratified According to their Presenting Syndromes. Chest 1997; 112:974-79. 13. Stein PD, Terrin ML, Hales CA, et al. Clinical, Laboratory Roentgenographic, and Electrocardiographic Findings in Patients with Acute Pulmonary Embolism and no Preexisting Cardiac or Pulmonary Disease. Chest 1991; 100: 598-603.

14. Perrier A1, Roy PM, Aujesky D, et al. Diagnosing pulmonary embolism in outpatients with clinical assessment, D-dimer measurement, venous ultrasound, and helical computed tomography: a multicenter management study. Am J Med 2004 Mar 1;116(5):291-9.

15. Elliott CG, Goldhaber SZ, Jensen RL. Delays in Diagnosis of Deep Vein Thrombosis and Pulmonary Embolism. Chest 2005; 128(5): 3372-76.

16. Hoo GW, Wu CC, Vazirani S, Borack BM. Does a Clinical Decision Rule using D-Dimer Levels Improve the Yield of CT Angiography? AJR 2011; 196(5): 1059-64.

17. Penaloza A, Verschuren F, Meyer G, Quentin-Georget S, Soulie C, Thys F, and Roy PM. Comparison of the unstructured clinician gestalt, the Wells score, and the revised Geneva score to estimate pretest probability for suspected pulmonary embolism. Ann Emerg Med 2013 Aug; 62(2):117124.e2.

18. Moores LK, King CS, Holley AB. Current Approach to the Diagnosis of Acute Nonmassive Pulmonary Embolism. Chest 2011; 140(2): 509-18.

19. Ceriani NS, Sohne M, Kruip MJ, et al. Christopher Study Investigators. Further Validation and Simplification of the Wells Clinical Decision Rule in Pulmonary Embolism. Thromb Haemost 2008; 99(1): 229-34.

20. Douma RA, Gibson NS, Gerdes VE, et al. Validity and Clinical Utility of the Simplified Wells Rule for assessing Clinical Probability for the Exclusion of Pulmonary Embo- lism. Thromb Haemost. 2009; 101(1): 197-200.

21. Le Gal G, Righini M, Roy PM, et al. Prediction of Pulmonary Embolism in the Emergency Department: the Revised Geneva Score. Ann Intern Med 2006; 144 (3): 165-71. 22. Klok FA, Kruisman E, Spaan J, et al. Comparison of the revised Geneva Score with the Wells Rule for assessing clinical probability of pulmonary embolism. J Thromb Haemost 2008; 6: 40-4.

23. Ceriani E, Combescure C, Le Gal G, et al. Clinical Prediction Rules for Pulmonary Embolism: a systematic review and meta-analysis. J Thromb Haemost 2010; 8:957-70.

24. Douma RA, Mos IC, Erkens PM, et al. Performance of 4 Clinical Decision Rules in the diagnostic management of acute pulmonary embolism: A prospective cohort study. Ann Intern Med 2011; 154(11): 709-18.

25. Wells PS, Anderson DR, Rodger M, et al. Derivation of a simple clinical model to categorize patients probability of pulmonary embolism: increasing the models utility with the SimpliRED D-dimer. Thromb Haemost 2000 Mar: 83(3); 416-20.

26. Bokobza J, Aubry A, Nakle N et al. Pulmonary Embolism Rule-out Criteria vs D-dimer testing in low-risk patients for pulmonary embolism: a retrospective study. Am J Emerg Med 2014 Jun; 32(6):609-13.

27. O'Connor C, Moriarty J, Walsh J, et al. The Application of a Clinical Risk Stratification Score may Reduce Unnecessary Investigations for Pulmonary Embolism in Pregnancy. J Matern Fetal Neonatal Med 2011; 24(12):1461-64.

28. Elliot CG, Goldhaber SZ, Visani L, De Rosa M. Chest Radiographs in Acute Pulmonary Embolism. Chest 2000; 118: 33-8.

29. Elliott CG, Goldhaber SZ, Visani L, DeRosa M. Chest Radiographs in Acute Pulmonary Embolism: Results from the International Cooperative Pulmonary Embolism Registry. Chest 2000; 118:33-38.

30. Panos RJ, Barish RA, Whye DW Jr, Groleau G. The electrocardiographic manifestations of pulmonary embolism. J Emerg Med 1988; 6:301.

31. Geibel A, Zehender M, Kasper W, Olschewski M, Klima C, Konstantinides SV. Prognostic value of the ECG on admission in patients with acute major pulmonary embolism. Eur Respir J 2005; 25:843-848.

32. Söhne M, ten Wolde M, Boomsma F, Reitsma JB, Douketis JD, Büller HR. Brain natriuretic peptide in hemodynamically stable acute pulmonary embolism. J Thromb Haemost 2006; 4:552-6.

33. Meyer T, Binder L, Hruska N, Luthe H, Buchwald AB. Cardiac Troponin I Elevation in Acute Pulmonary Embolism is Associated with Right Ventricular Dysfunction. J Am Coll 
Cardiol 2000; 36:1632-6.

34. Ruan LB, He L, Zhao S, et al. Prognostic value of plasma Heart-type Fatty Acid Binding Protein in patients with acute Pulmonary Embolism: a Meta-analysis. Chest 2014 Jul 3. doi: 10.1378/chest.13-1008.

35. Bruinstroop E, van de Ree MA, Huisman MV. D-dimer in specific clinical conditions: a narrative review. Eur J Intern Med. 2009; 20(5):441-46.

36. Brotman DJ, Segal JB, Jani JT, et al. Limitations of Ddimer Testing in Unselected Patients with Suspected Venous Thromboembolism. Am J Med 2003; 114(4): 276-82.

37. Wells PS, Anderson DR, Rodger MA, Stiell M, et al. Excluding Pulmonary Embolism at the bedside without Diagnostic Imaging: Management of Patients with Suspected Pulmonary Embolism Presenting to the Emergency Deparment by Using a Simple Clinical Model and d-dimer. Ann Intern Med 2001; 135:98-107.

38. Stein PD, Hull RD, Patel KC, et al. D-Dimer for the Exclusion of Acute Venous Thrombosis and Pulmonary Embolism: A systematic review. Ann Intern Med 2004; 140(8): 589-602.

39. Righini M, Van Es J, Den Exter PL, et al. Age-adjusted D-dimer cutoff levels to rule out pulmonary embolism: the ADJUST-PE study. JAMA 2014 Mar 19; 311(11):1117-24.

40. Pasha SM, Klok FA, Snoep JD, et al. Safety of Excluding Acute Pulmonary Embolism based on an Unlikely Clnical Probability by the Wells rule and normal D-dimer concentration: A meta-analysis Thromb Res 2010; 125(4):e123-27. 41. Kruip, MJ, Sohne M, Nijkeuter M, et al. Christopher Study Investigators. Accuracy of Clinical decision rule, D-dimer, and spiral-computed tomography in patients with malignancy, previous venous thromboembolism, COPD, or heart failure in older patients with suspected PE. J Thrombo Haemost 2006; 260(5):459-66.

42. Salaun PY, Couturaud F, Le Duc-Pennec, A, et al. Noninvasive Diagnosis of Pulmonary Embolism. Chest 2011; 139(6):1294-98.

43. Gibson NS, Sohne M, Gerdes VEA, et al. The Clinical of Clinical Probability Assessment in Interpreting a Normal d-Dimer in Patients With Suspected Pulmonary Embolism. Chest 2008; 134: 789-93.

44. Kruip JHA, Slob MJ, Schijen JHE, et al. Use of a Clinical Decision Rule in Combination with D-Dimer Concentration in Diagnostic Workup of Patients with Suspected Pulmonary Embolus. Arch Intern Med 2002; 162: 1631-35.

45. Le Gal F, Righini M, Sanchez O, et al. A Positive Compression Ultrasonography of the Lower Limb Veins is Highly Predicitive of Pulmonary Embolism on Computed Tomography in Suspected Patients. Thromb Hemost 2006; 95(6): 963-66.
46. Turkstra F, Kuijer PM, van Beek EJ, et al. Diagnostic Utility of ultrasonograpy of leg veins in patients with suspected pulmonary embolism. Ann Intern Med 1997; 126(10):775-81. 47. Sheiman RG, McArdle CR. Clinically Suspected Pulmonary Embolism: Use of bilateral lower extremity US as the initial examination-a prospective study. Radiology 1999; 212(1): 75-8.

48. PIOPED Investigators. Value of the Ventilation-Perfusion Lung Scan in Acute Pulmonary Embolism. JAMA 1990; 263(20):2753-2759.

49. Roach PJ, Schembri GP, Bailey DL. V/Q scanning using SPECT and SPECT/CT. J Nucl Med 2013 Sep; 54(9):158896.

50. Soler X, Hoh CK, Test VJ, et al. Single photon emission computed tomography in chronic thromboembolic pulmonary hypertension. Respirology 2011 Jan; 16(1):131-7.

51. Anderson DR, Kahn SR, Rodger MA, et al. Computed Tomographic Angiography versus Ventilation-Perfusion Lung Scanning in Patients with Suspected Pulmonary Embolism: a randomized controlled trial. JAMA 2007; 298(23)2743-53. 52. Sostman HD, Miniati M, Gottschalk A, et al. Sensitivity and Specificity of Perfusion Scintigraphy Combined with Chest Radiography for Acute Pulmonary Embolism in PIOPED II. J Nuc Med 2008; 49: 1741-48.

53. PIOPED II INVESTIGATORS. Multidectector Computed Tomography for Acute Pulmonary Embolism. N Engl J Med 2006; 354: 2317-27.

54. Feng LB1, Pines JM, Yusuf HR, et al. U.S. trends in computed tomography use and diagnoses in emergency department visits by patients with symptoms suggestive of pulmonary embolism, 2001-2009. Acad Emerg Med. 2013 Oct; 20(10):1033-40.

55. Sarma A, Heilbrun ME, Conner KE, et al. Radiation and chest CT scan examinations: what do we know? Chest 2012 Sep; 142(3):750-60. doi: 10.1378/chest.11-2863.

56. Estrada-Y-Martin RM, Oldham SA. CTPA as the gold standard for the diagnosis of pulmonary embolism. Int J Comut Assist Radiol Surger 2011; 6(4):557-63.

57. Stein PD, Athanasoulis C, Alavi A, et al. Complications and validitiy of pulmonary angiography in acute pulmonary embolism. Circulation1992; 85 (2):462-68.

58. Stein PD, Afzal A, Henry JW, Villareal CG. Fever in Pulmonary Embolism. Chest 2000; 117:39-42.

59. Winer-Muram HT, Rydberg J, Johnson MS, et al. Suspected Acute Pulmonary Embolism: Evaluation with Multidetector CT versus digital subtraction pulmonary angiography. Radiology 2004; 233 (3): 806-815.

60. Wittram C, Waltman AC, Shepherd JA, Halpern E, et al. Discordance between CT and Angiography in the PIOPED II Study. Radiology 2007; 244(3):883-89. 
61. Stein PD, Chenevert TL, Fowler SE, et al. Gadolinium enhanced magnetic resonance angiography for pulmonary embolism: a multicenter prospective study (PIOPED III). Ann Intern Med 2010; 152(7):434-43.

62. Nazerian P, Vanni S, Volpicelli G, et al. Accuracy of point-of-care multiorgan ultrasonography for the diagnosis of pulmonary embolism. Chest 2014 May; 145(5):950-7.

63. Stein PD, Janjua M, Matta F, et al. Prognosis effect of CK $-\mathrm{MB}$, Troponin I, RV enlargement seen on echocardiogram in stable patients with acute PE. AM J Cardio 107(5): 774-7.

64. Mookadem F, Jiaspirong P, Goel R, et al. Critical Appraisal on the Utility of Echocardiography in the Management of Acute Pulmonary Embolism. Cardio Rev 2011. 18(1):29-37. 65. Praszczyk KP, Torbicki A, Pacho R, et al. Noninvasive diagnosis of suspected severe acute Pulmonary Embolism: Transesophageal Echocardiography versus Spiral CT angiography. Chest 1997; 11 (3): 722-8. 